\title{
CHEMISTRY OF BLEOMYCIN. XVIII. CARBON-13 NMR STUDIES
}

\author{
Hiroshi Naganawa, Yasuhiko Muraoka*, Tomohisa Takita \\ and HAMAO UMEZAWA \\ Institute of Microbial Chemistry, Kamiosaki, Shinagawa-ku, Tokyo 141, Japan \\ *Research Laboratory, Nippon Kayaku Co. Ltd., \\ Shimo, Kita-ku, Tokyo 115, Japan
}

(Received for publication February 16, 1977)

\begin{abstract}
During chemical studies of bleomycin, many fragments and derivatives have been isolated and characterized. The ${ }^{18} \mathrm{C}$-NMR spectra of these compounds were taken and analyzed for structural information, and the complete assignment of the spectra was achieved. The ${ }^{13} \mathrm{C}$-chemical shift map thus obtained contains information about the structure and conformation and will be useful for studies on the chemistry and biology of bleomycin and related compounds.
\end{abstract}

The ${ }^{13} \mathrm{C}$-NMR spectroscopic study of bleomycin (BLM) has played an important role in the final stage of determination of the total structure of $\mathrm{BLM}^{11}$. That is: the ${ }^{13} \mathrm{C}-\mathrm{NMR}$ spectrum indicated the exact carbon number present in the molecule of BLM and gave information about their bonding character.

During the lengthy chemical studies of BLM, we have isolated many degradation products including amino acid components, terminal amines, sugar components and peptide fragments; also various minor modification products of BLM have been isolated and characterized. A collection of the ${ }^{13} \mathrm{C}$ NMR spectra of these well-characterized and related compounds has been analyzed for their structural relations with reference to known chemical shift data and established chemical shift rules and each signal has been assigned to a specific carbon atom in the BLM molecule. Some ambiguities were solved by the aid of single frequency ${ }^{1} \mathrm{H}$-decoupling.

\section{Materials and Methods}

The ${ }^{13} \mathrm{C}$-NMR spectra were taken with a Varian XL-100 FT NMR spectrometer at $25.2 \mathrm{MHz}$ at ambient temperature using $\mathrm{D}_{2} \mathrm{O}$ as a solvent in 8 -mm tubes. The samples $(50 \sim 80 \mu$ moles $)$ were dissolved in about $0.6 \mathrm{ml}$ of the solvent, and the pDs were adjusted to $6.0 \sim 6.5$, except for ring-expanded BLM A2'-c (vide post), $\mathrm{pD}$ of which was adjusted to 7.5 to prevent decomposition during the measurement. The measurement conditions were as follows: spectral width, $5120 \mathrm{~Hz}$; pulse flipping angle, $30 \sim 45^{\circ}$; aquisition time, $0.8 \mathrm{sec}$; number of data points, 8192 ; number of transients, $1 \times 10^{4} \sim 7 \times 10^{4}$. Dioxane was used as an internal reference of ${ }^{13} \mathrm{C}$-chemical shift, which was adjusted to $\delta$ 67.4. The ${ }^{1} \mathrm{H}$ chemical shift was also expressed as $\delta$ and external tetramethylsilane was used as the standard.

The degradation products measured were as follows: Amino acid components $\mathrm{I} \sim \mathrm{VI}^{2)}$ (for the structures and numbering, see Fig. 1), sugar components (methyl- $\alpha$-D-mannopyranoside and its 2 - and 3-O-carbamoyl derivatives ${ }^{3,4)}$ and methyl- $\alpha$-L-gulopyranoside ${ }^{3)}$ ), and peptide fragments (pseudotripeptide, pseudotetrapeptide $A^{5)}$, tripeptide $S^{51}$ and tetrapeptide $S^{6)}$, for the structures, see Fig. 2).

The minor modification products of BLM measured were as follows: Bleomycinic acid (BLMacid) ${ }^{7)}$, deglyco-BLM-acid (BLM-acid lacking sugar part, isolated from a culture filtrate of BLM fermentation, unpublished), decarbamoyl BLM A2'-c (formed during storage of BLM A2'-c in a Trisbuffer of $\mathrm{pH} 8.5$ at room temperature, unpublished), iso-BLM $\mathrm{A} 2^{\prime}-\mathrm{c}^{8)}$, epi-BLM $\mathrm{A} 2^{\prime}-\mathrm{c}^{91}$, deamide BLM A2 $-\mathrm{c}^{10)}$, ring-expanded BLM A2 $-\mathrm{c}^{11)}$ and depyruvamide BLM A2 $-\mathrm{c}^{11}$, of which structures are shown in Fig. 3. 


\section{Results and Discussion}

The chemical shifts of the ${ }^{13} \mathrm{C}-\mathrm{NMR}$ spectra of all measured samples except for monosaccharide derivatives are tabulated in Table 1.

The broad band ${ }^{1} \mathrm{H}$-decoupled ${ }^{13} \mathrm{C}$-NMR spectrum of BLM A2'-c is shown in Fig. 4. There were $24 \mathrm{sp}^{2}$-carbon signals in the $\delta 110 \sim 180$ range. In the ${ }^{1} \mathrm{H}$-off-resonance-decoupled ${ }^{13} \mathrm{C}$ NMR spectrum six signals, marked by $d$ in Fig. 4, appeared as doublet and others were singlet in this $\mathrm{sp}^{2}$-carbon region. Two signals assigned to the $\mathrm{C}-1$ of sugars appeared at about $\delta 100$, isolated from the other $\mathrm{sp}^{3}$-carbon signals. There were 27 peaks of $\mathrm{sp}^{3}$-carbons in the $\delta 10 \sim 80$ range, in which there were two overlapped peaks at $\delta 67.9$ and 75.2. In the ${ }^{1} \mathrm{H}$-off-resonancedecoupled spectrum the upper four peaks without mark in Fig. 4 were quartet, eight signals in
Fig. 1. Amino acid components of bleomycin.

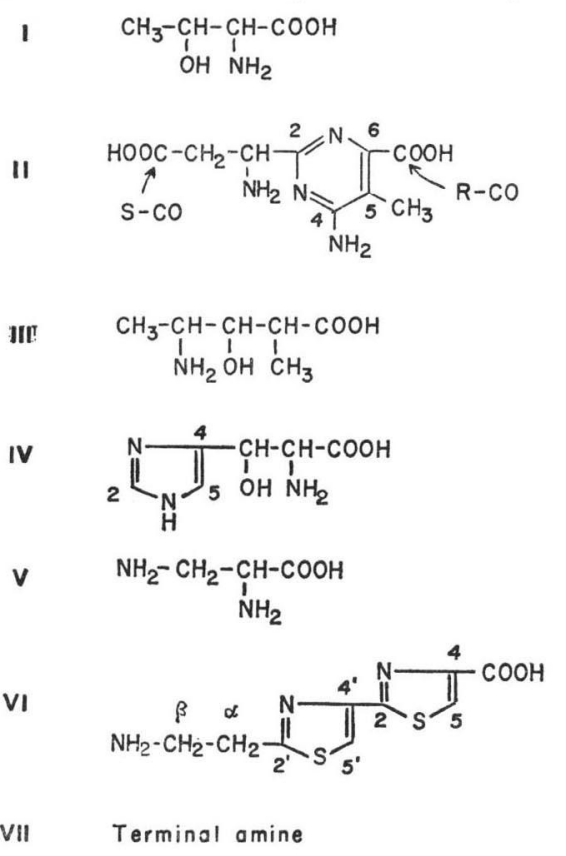

Fig. 2. Peptide fragments of bleomycin A2.

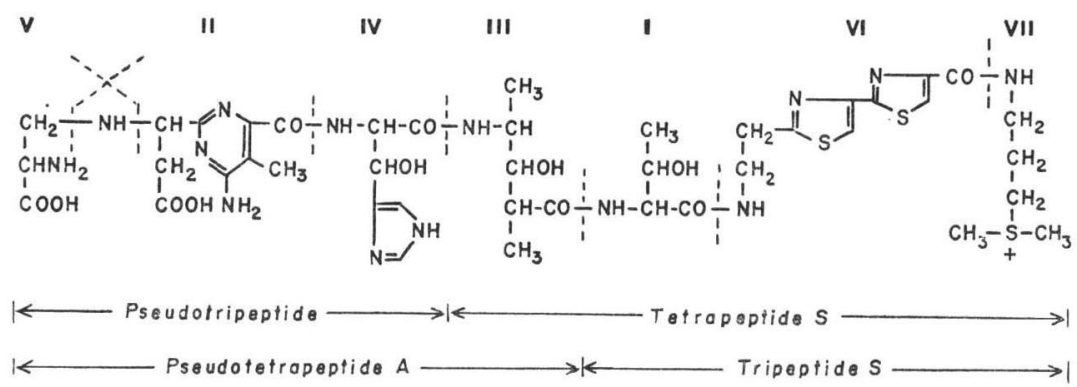

the middle with mark $t$ were triplet, the lower 15 peaks without mark were doublet and there was no singlet signal in this range.

\section{Terminal Amines}

The signals derived from the terminal amines were easily recognized by comparison of the spectra of BLM A2 and A2'-c (see Table 2). These BLMs were different from each other in their terminal amines, both containing five carbon atoms, and their spectra were different in five signals which could not be superimposed. These signals were naturally assigned to the carbons of the terminal amines, and each signal could be assigned as shown in Table 1. Table 2 indicates that the chemical shifts of the common part of the two BLMs were the same within $\pm 0.1 \mathrm{ppm}$, except for two signals which will be discussed later. In the following discussions, the chemical shift values of BLM A2'-c are mainly used.

\section{C-Methyl Group}

There are four $\mathrm{C}-$ methyl groups. They were assigned to $\mathrm{I}-\mathrm{CH}_{3}(\delta 19.9), \mathrm{III}-\alpha-\mathrm{CH}_{3}$ (15.8), III- 
Table $1 .{ }^{13} \mathrm{C}$-Chemical shift map of bleomycin

\begin{tabular}{|c|c|c|c|c|c|c|c|c|c|c|c|c|c|c|c|c|}
\hline & 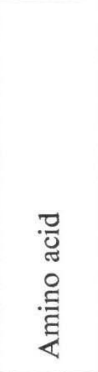 & 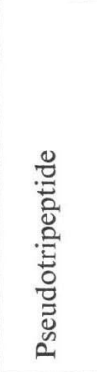 & 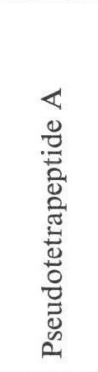 & 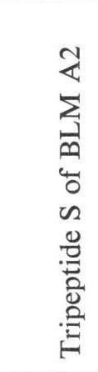 & 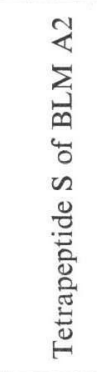 & 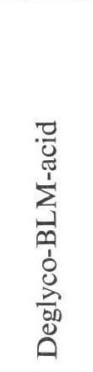 & 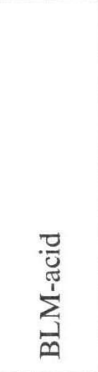 & $\begin{array}{l}\frac{y}{d} \\
\sum \\
\bar{x}\end{array}$ & $\begin{array}{l}0 \\
i \\
i \\
2 \\
\sum \\
0\end{array}$ & 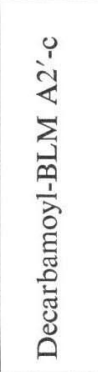 & $\begin{array}{l}0 \\
i \\
2 \\
\Sigma \\
\Sigma \\
0 \\
0 \\
0 \\
0 \\
0\end{array}$ & 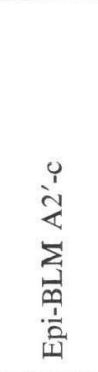 & 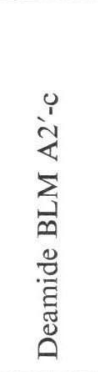 & 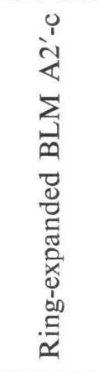 & 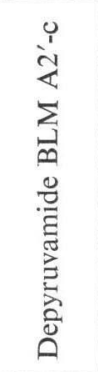 & 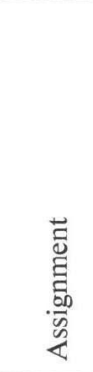 \\
\hline I & $\begin{array}{r}175.0 \\
66.7 \\
61.1 \\
19.3\end{array}$ & & & $\begin{array}{r}168.6 \\
66.9 \\
59.6 \\
19.8\end{array}$ & $\begin{array}{r}172.3 \\
67.9 \\
60.0 \\
19.9\end{array}$ & $\begin{array}{r}172.5 \\
67.9 \\
59.9 \\
19.9\end{array}$ & $\begin{array}{r}172.5 \\
67.9 \\
60.0 \\
19.9\end{array}$ & \begin{tabular}{|r|}
172.5 \\
68.0 \\
59.9 \\
20.0
\end{tabular} & $\begin{array}{r}172.5 \\
67.9 \\
59.9 \\
19.9\end{array}$ & $\begin{array}{r}172.5 \\
67.9 \\
59.9 \\
19.9\end{array}$ & $\begin{array}{r}172.5 \\
67.9 \\
59.9 \\
19.9\end{array}$ & $\begin{array}{r}172.5 \\
68.0 \\
59.9 \\
20.0\end{array}$ & $\begin{array}{r}172.5 \\
67.9 \\
59.9 \\
19.9\end{array}$ & $\begin{array}{r}172.5 \\
68.0 \\
59.9 \\
20.0\end{array}$ & $\begin{array}{r}172.5 \\
67.9 \\
59.9 \\
19.9\end{array}$ & $\begin{array}{l}\mathrm{CO} \\
\beta-\mathrm{CH} \\
\alpha-\mathrm{CH} \\
\mathrm{CH}_{3}\end{array}$ \\
\hline II & $\begin{array}{r}172.8 \\
166.6 \\
157.1 \\
165.1 \\
146.0 \\
114.5 \\
49.5 \\
36.9 \\
12.3\end{array}$ & $\begin{array}{r}177.2 \\
167.8 \\
160.4 \\
165.4 \\
153.2 \\
113.6 \\
50.8 \\
38.5 \\
11.9\end{array}$ & $\begin{array}{r}177.4 \\
168.0 \\
161.1 \\
165.3 \\
153.2 \\
113.3 \\
51.1 \\
38.7 \\
11.9\end{array}$ & & & $\begin{array}{r}176.7 \\
168.1 \\
165.9 \\
164.8 \\
153.7 \\
112.2 \\
53.1 \\
40.9 \\
11.8\end{array}$ & $\begin{array}{r}176.8 \\
168.2 \\
165.7 \\
165.1 \\
152.8 \\
113.0 \\
53.2 \\
41.0 \\
11.9\end{array}$ & $\begin{array}{r}176.7 \\
168.3 \\
165.9 \\
165.2 \\
152.9 \\
113.0 \\
53.3 \\
41.0 \\
11.9\end{array}$ & $\begin{array}{r}176.7 \\
168.3 \\
165.9 \\
165.2 \\
152.9 \\
113.0 \\
53.3 \\
41.1 \\
11.8\end{array}$ & $\begin{array}{r}176.7 \\
168.3 \\
166.1 \\
164.9 \\
153.9 \\
112.3 \\
53.4 \\
41.1 \\
11.8\end{array}$ & $\begin{array}{r}176.7 \\
168.5 \\
166.3 \\
164.9 \\
154.0 \\
112.2 \\
53.6 \\
41.1 \\
11.8\end{array}$ & $\begin{array}{r}176.3 \\
168.3 \\
166.1 \\
165.6 \\
151.3 \\
114.3 \\
53.7 \\
42.0 \\
11.9\end{array}$ & $\begin{array}{r}176.6 \\
168.3 \\
165.5 \\
165.2 \\
153.0 \\
112.9 \\
54.5 \\
40.7 \\
11.9\end{array}$ & $\begin{array}{r}178.8 \\
168.1 \\
165.1 \\
165.1 \\
152.2 \\
113.3 \\
56.7 \\
44.8 \\
11.9\end{array}$ & $\begin{array}{r}174.5 \\
168.0 \\
160.6 \\
165.4 \\
152.0 \\
114.1 \\
52.5 \\
37.1 \\
11.9\end{array}$ & $\begin{array}{l}\text { S-CO } \\
\text { R-CO } \\
2 \\
4 \\
6 \\
5 \\
\mathrm{CH} \\
\mathrm{CH}_{2} \\
\mathrm{CH}_{3}\end{array}$ \\
\hline III & $\begin{array}{r}182.7 \\
73.6 \\
50.5 \\
47.0 \\
16.0 \\
11.7\end{array}$ & & $\begin{array}{r}182.6 \\
75.6 \\
48.8 \\
45.2 \\
15.6 \\
13.4\end{array}$ & & $\begin{array}{r}177.0 \\
72.9 \\
50.1 \\
44.2 \\
15.9 \\
11.9\end{array}$ & $\begin{array}{r}178.0 \\
75.0 \\
48.6 \\
43.9 \\
15.0 \\
13.8\end{array}$ & $\begin{array}{r}178.1 \\
75.2 \\
48.5 \\
43.6 \\
15.7 \\
13.0\end{array}$ & \begin{tabular}{|r|}
178.1 \\
75.2 \\
48.5 \\
43.5 \\
15.8 \\
12.9
\end{tabular} & $\begin{array}{r}178.1 \\
75.2 \\
48.4 \\
43.5 \\
15.8 \\
12.9\end{array}$ & $\begin{array}{r}178.1 \\
75.2 \\
48.3 \\
43.5 \\
16.0 \\
12.9\end{array}$ & $\begin{array}{r}178.1 \\
75.2 \\
48.3 \\
43.5 \\
16.0 \\
12.8\end{array}$ & $\begin{array}{r}178.0 \\
75.3 \\
48.4 \\
43.6 \\
15.8 \\
12.8\end{array}$ & $\begin{array}{r}178.1 \\
75.2 \\
48.4 \\
43.6 \\
15.7 \\
12.9\end{array}$ & $\begin{array}{r}178.1 \\
75.1 \\
48.4 \\
43.5 \\
15.9 \\
12.8\end{array}$ & $\begin{array}{r}178.0 \\
75.2 \\
48.5 \\
43.6 \\
15.6 \\
13.0\end{array}$ & $\begin{array}{l}\mathrm{CO} \\
\beta-\mathrm{CH} \\
\gamma-\mathrm{CH} \\
\alpha-\mathrm{CH} \\
\alpha-\mathrm{CH}_{3} \\
\gamma-\mathrm{CH}_{3}\end{array}$ \\
\hline IV & $\begin{array}{r}170.4 \\
135.7 \\
132.2 \\
117.3 \\
64.6 \\
59.6\end{array}$ & $\begin{array}{r}174.7 \\
134.4 \\
133.2 \\
117.2 \\
66.9 \\
59.5\end{array}$ & $\begin{array}{r}169.4 \\
135.1 \\
133.4 \\
117.7 \\
65.8 \\
58.4\end{array}$ & & & $\begin{array}{r}169.5 \\
135.9 \\
134.9 \\
117.6 \\
66.5 \\
58.4\end{array}$ & $\begin{array}{r}169.3 \\
137.3 \\
134.4 \\
118.9 \\
68.6 \\
57.6\end{array}$ & $\begin{array}{r}169.4 \\
137.4 \\
134.7 \\
118.6 \\
68.6 \\
57.7\end{array}$ & $\begin{array}{r}169.5 \\
137.5 \\
135.0 \\
118.5 \\
68.6 \\
57.7\end{array}$ & $\begin{array}{r}169.7 \\
137.6 \\
135.6 \\
118.5 \\
68.5 \\
57.5\end{array}$ & $\begin{array}{r}169.7 \\
137.7 \\
135.6 \\
118.4 \\
67.9 \\
57.4\end{array}$ & $\begin{array}{r}169.4 \\
137.8 \\
135.4 \\
117.9 \\
68.0 \\
57.3\end{array}$ & $\begin{array}{r}169.4 \\
137.2 \\
134.2 \\
118.7 \\
68.5 \\
57.7\end{array}$ & $\begin{array}{r}169.9 \\
137.7 \\
136.3 \\
118.4 \\
68.6 \\
57.9\end{array}$ & $\begin{array}{r}169.2 \\
136.9 \\
133.9 \\
118.8 \\
68.6 \\
57.4\end{array}$ & $\begin{array}{l}\mathrm{CO} \\
2 \\
4 \\
5 \\
\beta-\mathrm{CH} \\
\alpha-\mathrm{CH}\end{array}$ \\
\hline
\end{tabular}




\begin{tabular}{|c|c|c|c|c|c|c|c|c|c|c|c|c|c|c|c|}
\hline V & $\begin{array}{r}171.6 \\
51.1 \\
39.7 \\
\end{array}$ & $\begin{array}{r}171.8 \\
60.7 \\
46.1\end{array}$ & $\begin{array}{r}171.9 \\
60.7 \\
46.1 \\
\end{array}$ & & $\begin{array}{r}171.6 \\
60.5 \\
47.8\end{array}$ & $\begin{array}{r}171.6 \\
60.5 \\
47.9 \\
\end{array}$ & $\begin{array}{r}171.6 \\
60.5 \\
47.8\end{array}$ & $\begin{array}{r}171.6 \\
60.4 \\
47.8\end{array}$ & $\begin{array}{r}172.1 \\
60.5 \\
48.2\end{array}$ & $\begin{array}{r}172.1 \\
60.4 \\
48.3\end{array}$ & $\begin{array}{r}171.5 \\
61.5 \\
48.1\end{array}$ & $\begin{array}{r}173.3 \\
60.5 \\
47.3\end{array}$ & $\begin{array}{r}174.6 \\
58.1 \\
49.9\end{array}$ & & $\begin{array}{l}\mathrm{CO} \\
\mathrm{CH} \\
\mathrm{CH}_{2}\end{array}$ \\
\hline VI & $\begin{array}{r}170.4 \\
164.1 \\
161.3 \\
142.6 \\
142.4 \\
131.2 \\
126.0 \\
39.3 \\
30.3\end{array}$ & & $\begin{array}{r}170.9 \\
163.6 \\
162.9 \\
149.3 \\
147.5 \\
125.7 \\
119.8 \\
40.3 \\
32.7\end{array}$ & $\begin{array}{r}171.3 \\
163.9 \\
163.2 \\
149.4 \\
147.6 \\
125.8 \\
119.8 \\
40.1 \\
32.9\end{array}$ & $\begin{array}{r}170.9 \\
168.9 \\
162.2 \\
154.1 \\
148.0 \\
125.6 \\
118.9 \\
39.9 \\
32.8\end{array}$ & $\begin{array}{r}170.8 \\
169.0 \\
162.2 \\
154.1 \\
147.9 \\
125.6 \\
118.9 \\
40.0 \\
32.8\end{array}$ & $\begin{array}{r}171.0 \\
163.7 \\
163.0 \\
149.4 \\
147.5 \\
125.7 \\
119.8 \\
40.0 \\
32.9\end{array}$ & $\begin{array}{r}170.9 \\
163.4 \\
162.9 \\
149.4 \\
147.5 \\
125.6 \\
119.7 \\
40.0 \\
32.8\end{array}$ & $\begin{array}{r}170.9 \\
163.3 \\
162.9 \\
149.4 \\
147.5 \\
125.5 \\
119.6 \\
40.0 \\
32.9\end{array}$ & $\begin{array}{r}170.9 \\
163.3 \\
162.9 \\
149.4 \\
147.5 \\
125.5 \\
119.6 \\
40.0 \\
32.8\end{array}$ & $\begin{array}{r}171.0 \\
163.4 \\
163.0 \\
149.4 \\
147.6 \\
125.6 \\
119.7 \\
40.0 \\
32.9\end{array}$ & $\begin{array}{r}171.0 \\
163.4 \\
163.0 \\
149.4 \\
147.5 \\
125.7 \\
119.7 \\
40.0 \\
32.8\end{array}$ & $\begin{array}{r}170.7 \\
163.0 \\
162.6 \\
149.5 \\
147.5 \\
125.3 \\
119.5 \\
40.0 \\
32.9\end{array}$ & $\begin{array}{r}171.1 \\
163.6 \\
163.1 \\
149.4 \\
147.6 \\
125.7 \\
119.8 \\
40.0 \\
32.8\end{array}$ & $\begin{array}{l}\mathrm{CO} \\
2 \\
2^{\prime} \\
4 \\
4^{\prime} \\
5^{\prime} \\
5 \\
\beta-\mathrm{CH}_{2} \\
\alpha-\mathrm{CH}_{2}\end{array}$ \\
\hline $\begin{array}{l}\text { VII } \\
\text { (A2) }\end{array}$ & & & $\begin{array}{l}41.8 \\
38.5 \\
25.7 \times 2 \\
24.6\end{array}$ & $\begin{array}{l}41.8 \\
38.5 \\
25.7 \times 2 \\
24.6\end{array}$ & & & $\begin{array}{l}41.8 \\
38.6 \\
25.7 \times 2 \\
24.6\end{array}$ & & & & & & & & $\begin{array}{l}\alpha-\mathrm{CH}_{2} \\
\gamma-\mathrm{CH}_{2} \\
\mathrm{CH}_{3} \\
\beta-\mathrm{CH}_{2}\end{array}$ \\
\hline $\begin{array}{c}\text { VII } \\
\left(\mathrm{A} 2^{\prime}-\mathrm{c}\right)\end{array}$ & & & & & & & & $\begin{array}{r}134.1 \\
131.7 \\
117.1 \\
39.2 \\
25.4\end{array}$ & $\begin{array}{r}134.7 \\
132.6 \\
117.2 \\
39.4 \\
25.8\end{array}$ & $\begin{array}{r}134.7 \\
132.7 \\
117.2 \\
39.5 \\
25.9\end{array}$ & $\begin{array}{r}134.1 \\
131.8 \\
117.1 \\
39.2 \\
25.5\end{array}$ & $\begin{array}{r}134.0 \\
131.6 \\
117.1 \\
39.2 \\
25.3\end{array}$ & $\begin{array}{r}135.7 \\
134.9 \\
117.6 \\
40.0 \\
27.0\end{array}$ & $\begin{array}{r}133.8 \\
131.5 \\
117.1 \\
39.2 \\
25.3\end{array}$ & $\begin{array}{l}2 \\
4 \\
5 \\
\alpha-\mathrm{CH}_{2} \\
\beta-\mathrm{CH}_{2}\end{array}$ \\
\hline Gulose & & & & & & $\begin{array}{l}98.4 \\
74.4 \\
71.0 \\
70.0 \\
68.1 \\
61.2\end{array}$ & $\begin{array}{l}98.3 \\
74.4 \\
71.0 \\
70.0 \\
68.0 \\
61.2\end{array}$ & $\begin{array}{l}98.2 \\
74.4 \\
71.0 \\
69.9 \\
67.9 \\
61.2\end{array}$ & $\begin{array}{l}98.9 \\
74.3 \\
71.0 \\
69.9 \\
67.9 \\
61.0\end{array}$ & $\begin{array}{l}98.5 \\
74.2 \\
70.1 \\
69.9 \\
67.9 \\
61.0\end{array}$ & $\begin{array}{l}98.4 \\
74.7 \\
70.3 \\
70.2 \\
68.0 \\
61.5\end{array}$ & $\begin{array}{l}98.4 \\
74.4 \\
70.9 \\
69.9 \\
68.2 \\
61.2\end{array}$ & $\begin{array}{l}98.6 \\
74.4 \\
70.8 \\
70.0 \\
68.0 \\
61.2\end{array}$ & $\begin{array}{l}98.5 \\
74.4 \\
71.0 \\
69.9 \\
68.3 \\
61.3\end{array}$ & $\begin{array}{l}1 \\
2 \\
3 \\
4 \\
5 \\
6\end{array}$ \\
\hline Mannose & & & & & & $\begin{array}{r}158.5 \\
98.8 \\
75.2 \\
73.7 \\
69.2 \\
65.6 \\
61.8\end{array}$ & $\begin{array}{r}158.5 \\
98.8 \\
75.2 \\
73.6 \\
69.2 \\
65.6 \\
61.8\end{array}$ & $\begin{array}{r}158.5 \\
98.9 \\
75.2 \\
73.7 \\
69.1 \\
65.6 \\
61.8\end{array}$ & $\begin{array}{l}98.9 \\
70.2 \\
74.3 \\
71.4 \\
67.6 \\
61.9\end{array}$ & $\begin{array}{r}158.6 \\
96.2 \\
69.9 \\
73.9 \\
73.5 \\
67.7 \\
61.6\end{array}$ & $\begin{array}{r}158.1 \\
97.2 \\
75.1 \\
71.9 \\
69.0 \\
65.4 \\
61.7\end{array}$ & $\begin{array}{r}158.5 \\
98.7 \\
75.2 \\
73.5 \\
69.1 \\
65.6 \\
61.8\end{array}$ & $\begin{array}{r}158.5 \\
98.6 \\
75.1 \\
74.7 \\
69.2 \\
65.6 \\
61.8\end{array}$ & $\begin{array}{r}158.6 \\
98.9 \\
75.2 \\
73.2 \\
69.1 \\
65.5 \\
61.8\end{array}$ & $\begin{array}{l}\mathrm{CO} \\
1 \\
3 \\
5 \\
2 \\
4 \\
6\end{array}$ \\
\hline
\end{tabular}


Fig. 3. Bleomycins and their derivatives.

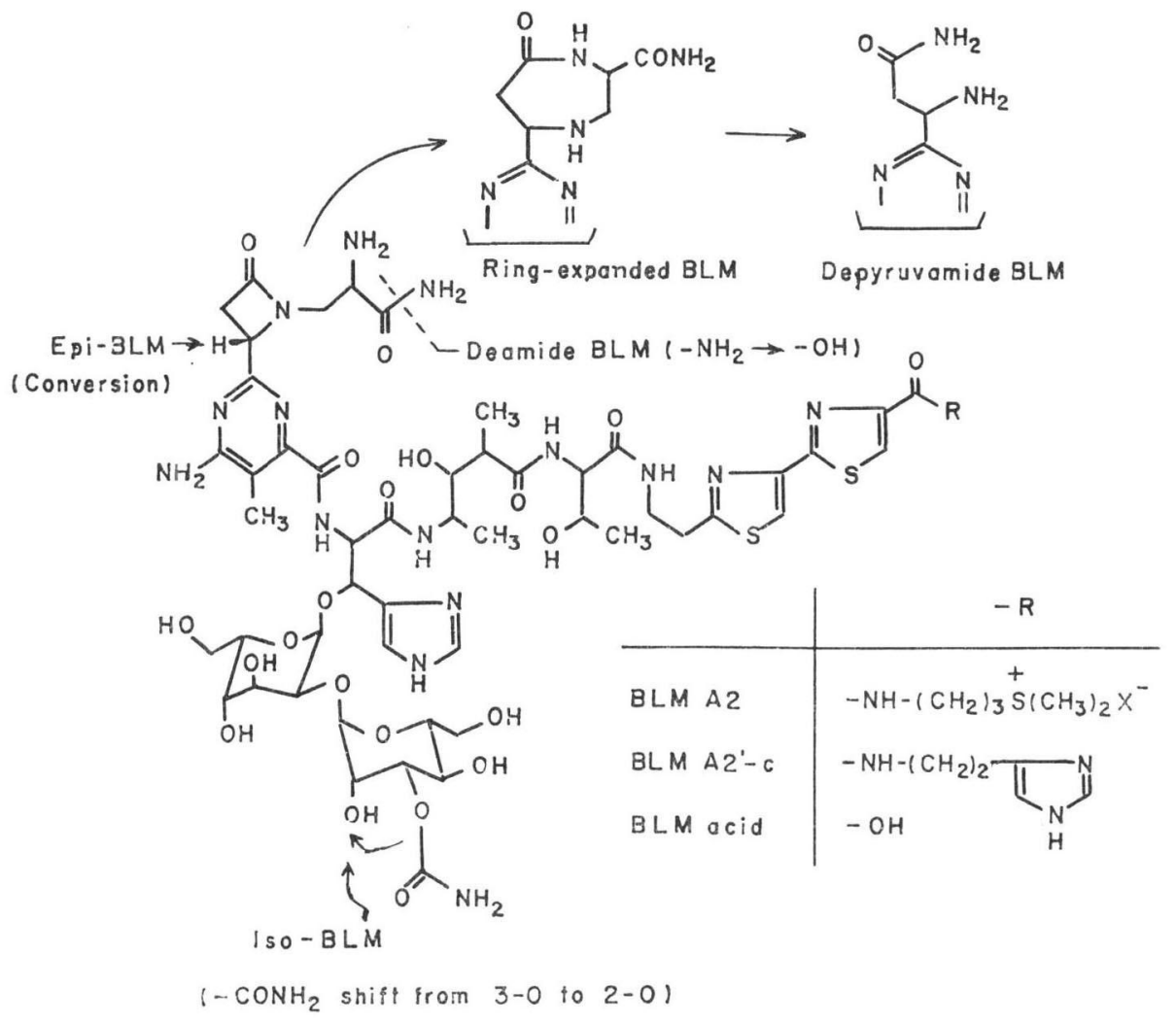

Fig. 4. The ${ }^{1} \mathrm{H}$-noise decoupled ${ }^{13} \mathrm{C}-\mathrm{NMR}$ spectrum of bleomycin $\mathrm{A} 22^{\prime}-\mathrm{c}$.

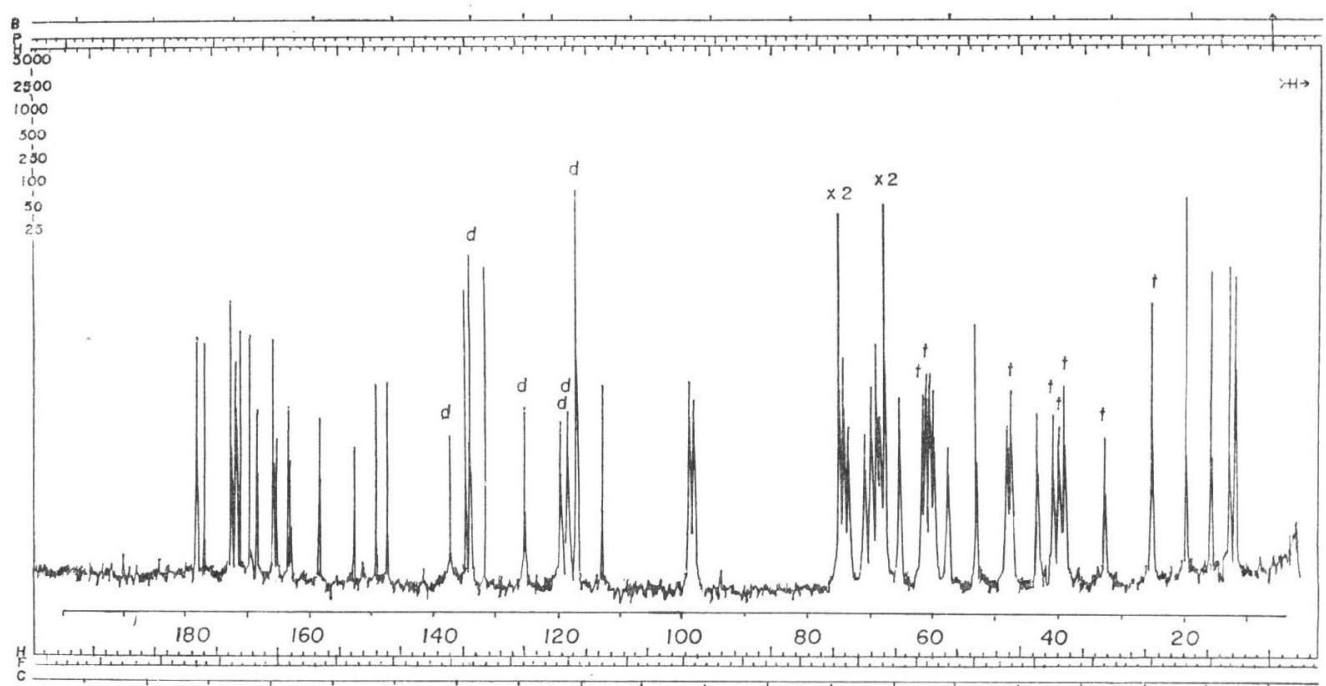

$\gamma-\mathrm{CH}_{3}$ (12.9) and II- $\mathrm{CH}_{3}$ (11.8), respectively, based on the spectra of the amino acids and peptide components. The assignment of $\mathrm{II}-\mathrm{CH}_{3}$ was confirmed by single frequency ${ }^{1} \mathrm{H}$-decoupling at $\delta$ 2.48, the chemical shift for the ${ }^{1} \mathrm{H}$ of $\mathrm{II}-\mathrm{CH}_{3}$ of $\mathrm{BLM} \mathrm{A} 2^{\prime}$-c. 
Table 2. ${ }^{13} \mathrm{C}$-Chemical shifts of bleomycins A2 and A2'-c

\begin{tabular}{c|c|c|c|c|c|c|c|c|c|c|c}
\hline $\mathrm{A} 2$ & $\mathrm{~A} 2^{\prime}-\mathrm{c}$ & $\Delta^{*}$ & $\mathrm{~A} 2$ & $\mathrm{~A} 2^{\prime}-\mathrm{c}$ & $\Delta$ & $\mathrm{A} 2$ & $\mathrm{~A} 2^{\prime}-\mathrm{c}$ & \multicolumn{1}{|c|}{$\Delta$} & $\mathrm{A} 2$ & $\mathrm{~A} 2^{\prime}-\mathrm{c}$ & $\Delta$ \\
\hline 178.1 & 178.1 & 0 & 137.4 & 137.5 & -0.1 & 71.0 & 71.0 & 0 & 43.5 & 43.5 & 0 \\
176.7 & 176.7 & 0 & 134.7 & 135.0 & -0.3 & 70.0 & 69.9 & 0.1 & 41.8 & - & - \\
172.5 & 172.5 & 0 & - & 134.1 & - & 69.2 & 69.1 & 0.1 & 41.0 & 41.1 & -0.1 \\
171.6 & 171.6 & 0 & - & 131.7 & - & 68.6 & 68.6 & 0 & 40.0 & 40.0 & 0 \\
171.0 & 170.9 & 0.1 & 125.7 & 125.6 & 0.1 & 68.0 & 67.9 & 0.1 & - & 39.2 & - \\
169.4 & 169.5 & -0.1 & 119.8 & 119.7 & 0.1 & 68.0 & 67.9 & 0.1 & 38.6 & - & - \\
168.3 & 168.3 & 0 & 118.6 & 118.5 & 0.1 & 65.6 & 65.6 & 0 & 32.9 & 32.8 & 0.1 \\
165.9 & 165.9 & 0 & - & 117.1 & - & 61.8 & 61.8 & 0 & 25.7 & - & - \\
165.2 & 165.2 & 0 & 113.0 & 113.0 & 0 & 61.2 & 61.2 & 0 & 25.7 & - & - \\
163.7 & 163.4 & 0.3 & 98.8 & 98.9 & -0.1 & 60.5 & 60.4 & 0.1 & - & 25.4 & - \\
163.0 & 162.9 & 0.1 & 98.3 & 98.2 & 0.1 & 59.9 & 59.9 & 0 & 24.6 & - & - \\
158.5 & 158.5 & 0 & 75.2 & 75.2 & 0 & 57.7 & 57.7 & 0 & 20.0 & 19.9 & 0.1 \\
152.9 & 152.9 & 0 & 75.2 & 75.2 & 0 & 53.3 & 53.3 & 0 & 15.8 & 15.8 & 0 \\
149.4 & 149.4 & 0 & 74.4 & 74.4 & 0 & 48.5 & 48.4 & 0.1 & 12.9 & 12.9 & 0 \\
147.5 & 147.5 & 0 & 73.6 & 73.7 & -0.1 & 47.8 & 47.8 & 0 & 11.9 & 11.8 & 0.1 \\
\hline
\end{tabular}

* Chemical shift difference (ppm) between $\mathrm{A} 2$ and $\mathrm{A} 22^{\prime}-\mathrm{c}$

\section{Sugar Part and Other Three Hydroxy-methines}

Two signals at $\delta 98.2$ and 98.9 and the two other signals at $\delta 61.2$ and 61.8 , both triplets by offresonance decoupling, could be easily assigned to the two $\mathrm{C}-1 \mathrm{~s}$ and two $\mathrm{C}-6 \mathrm{~s}$ of the disaccharide moiety apart from individual assignment. There were eleven signals in the $\delta 65.6$ to 75.2 range. The signals of the $\beta$-carbons of I and III could be assigned to $\delta 67.9$ and 75.2 respectively with reference to the spectra of pseudotetrapeptide A, tripeptide $\mathrm{S}$, tetrapeptide $\mathrm{S}$ and deglyco-BLM-acid. The signal of the $\beta$-carbon of IV was assigned to $\delta 68.6$ by single frequency ${ }^{1} \mathrm{H}$-decoupling irradiation of the $\beta$ - $\mathrm{CH}$ proton of IV, which resonated at $\delta 5.75$ and was lower than the other hydroxy-methine proton chemical shifts by at least $1.0 \mathrm{ppm}$.

The other eight signals between $\delta 65.6$ and 75.2 were due to the $\mathrm{C}-2$ to $\mathrm{C}-5$ of both sugars. In order to select the signals ascribed to the mannose moiety, the spectra of methyl- $\alpha$-D-mannopyranoside and its 2-O- and 3-O-carbamoyl derivatives were used as the reference compounds for the mannose

Table 3. ${ }^{13} \mathrm{C}$-Chemical shifts for $\alpha$-methylglycosides of mannose and its $\mathrm{O}$-carbamoyl derivatives and mannose moieties of bleomycins

\begin{tabular}{|c|c|c|c|c|c|c|c|c|}
\hline \multirow{2}{*}{ Compound } & \multicolumn{8}{|c|}{ Carbon } \\
\hline & $\mathrm{CO}$ & 1 & 2 & 3 & 4 & 5 & 6 & $\mathrm{CH}_{3}$ \\
\hline Me- $\alpha$-D-mannoside* (a) & & 101.6 & 71.4 & 70.8 & 67.6 & 73.4 & 61.8 & 55.7 \\
\hline $\begin{array}{l}\text { 3-O-Carb.-derivative (b) } \\
\text { (a)-(b) }\end{array}$ & 159.0 & $\begin{array}{l}101.4 \\
+0.2\end{array}$ & $\begin{array}{r}69.1 \\
+2.3\end{array}$ & $\begin{array}{r}75.2 \\
-4.4\end{array}$ & $\begin{array}{r}65.4 \\
+2.2\end{array}$ & $\begin{array}{c}73.4 \\
0\end{array}$ & $\begin{array}{r}61.7 \\
+0.1\end{array}$ & 55.6 \\
\hline $\begin{array}{l}\text { 2-O-Carb.-derivative (c) } \\
\text { (a)-(c) }\end{array}$ & 158.8 & $\begin{array}{r}99.2 \\
+2.4\end{array}$ & $\begin{array}{r}73.3 \\
-1.9\end{array}$ & $\begin{array}{r}69.9 \\
+0.9\end{array}$ & $\begin{array}{r}67.7 \\
-0.1\end{array}$ & $\begin{array}{r}73.3 \\
+0.1\end{array}$ & $\begin{array}{r}61.5 \\
+0.3\end{array}$ & 55.8 \\
\hline De-carb.-BLM A2'-c (d) & & 98.9 & 71.4 & 70.2 & 67.6 & 74.3 & 61.9 & \\
\hline $\begin{array}{l}\text { BLM A2'-c (e) } \\
(\text { d) }-(\mathrm{e})\end{array}$ & 158.5 & $\begin{array}{c}98.9 \\
0\end{array}$ & $\begin{array}{r}69.1 \\
+2.3\end{array}$ & $\begin{array}{r}75.2 \\
-5.0\end{array}$ & $\begin{array}{r}65.6 \\
+2.0\end{array}$ & $\begin{array}{r}73.7 \\
+0.6\end{array}$ & $\begin{array}{r}61.8 \\
+0.1\end{array}$ & \\
\hline $\begin{array}{l}\text { Iso-BLM A2'-c (f) } \\
\text { (d) -(f) }\end{array}$ & 158.6 & $\begin{array}{r}96.2 \\
+2.7\end{array}$ & $\begin{array}{r}73.5 \\
-2.1\end{array}$ & $\begin{array}{r}69.9 \\
+0.3\end{array}$ & $\begin{array}{r}67.7 \\
-0.1\end{array}$ & $\begin{array}{r}73.9 \\
+0.4\end{array}$ & $\begin{array}{r}61.6 \\
+0.3\end{array}$ & \\
\hline
\end{tabular}

\footnotetext{
* Assignment, according to A. S. PerLIN et al. ${ }^{12}$
} 
moieties of decarbamoyl-BLM, iso-BLM and BLM respectively. The results are shown in Table 3. With reference to the carbamoyl substitution effects, the signals of the carbons belonging to mannose moieties of decarbamoyl-BLM, iso-BLM and BLM could be selected and assigned to individual carbons as shown in Table 3. The assignment of C-1 was achieved by single frequency ${ }^{1} \mathrm{H}$-decoupling at $\delta 5.46$, at which the $\mathrm{C}_{1}-\mathrm{H}$ of the mannose moiety resonated while that of the gulose moiety resonated at $\delta 5.73$. The carbamoyl carbon could be also assigned to $\delta 158.5$ by these studies.

To assign the carbons of the gulose moiety, the spectrum of methyl- $\alpha$-L-gulopyranoside was taken as the reference and analyzed by single frequency irradiation of the $\mathrm{C}_{5}$-proton, because the chemical shift $(\delta 4.55)$ of this proton was separated only just enough from those of other protons $\left[\mathrm{C}_{1}-\mathrm{H}(5.27)\right.$, $\mathrm{C}_{2}-\mathrm{H}$ (4.41), $\mathrm{C}_{3}-\mathrm{H}$ (4.38), $\mathrm{C}_{4}-\mathrm{H}$ (4.32) and $\mathrm{C}_{6}-\mathrm{H}$ (4.22)] for single frequency ${ }^{1} \mathrm{H}$-decoupling. The assignment was in accord with the expected chemical shift estimated from the chemical shift of methyl- $\alpha$ galactopyranoside proposed by PerLin et al. ${ }^{12)}$ (Table 4). The signals of carbons belonging to the gulose moiety of BLM were assigned with reference to the chemical shift of this model compound and glycosidic shift-effect (Table 4).

Table 4. Assignment of ${ }^{18} \mathrm{C}$-chemical shift of gulose moiety of bleomycin estimated from that of galactose

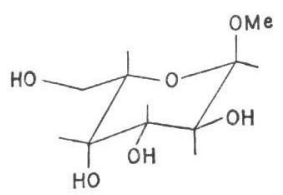

Me- $\alpha$-L-galactoside

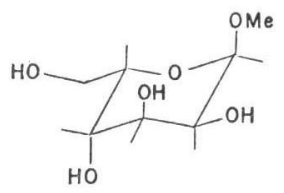

Me- $\alpha$-L-guloside

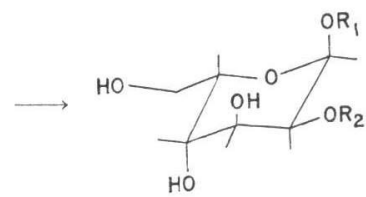

Gulose moiety of BLM

\begin{tabular}{|c|c|c|c|c|c|c|}
\hline Carbon & $\begin{array}{c}\text { Me- } \alpha \text {-L- } \\
\text { galactoside* }\end{array}$ & Interaction* & $\begin{array}{l}\text { Expected } \\
\text { shielding* }\end{array}$ & $\begin{array}{l}\text { Me- } \alpha \text {-L- } \\
\text { guloside }\end{array}$ & & $\begin{array}{l}\text { Gulose moiety } \\
\text { of bleomycin }\end{array}$ \\
\hline 1 & 100.5 & $\mathrm{O} / / \mathrm{O}-3,-\mathrm{O} / / \mathrm{H}-3$ &,+- & 100.4 & $\mathrm{OMe} \rightarrow \mathrm{OR}_{1}$ & 98.2 \\
\hline 2 & 70.6 & adj. 3, & + & 65.5 & $\mathrm{OH} \rightarrow \mathrm{OR}_{2}$ & 74.4 \\
\hline 3 & 70.9 & $\left\{\begin{array}{l}\text { ax. O, } \quad \mathrm{O} / / \mathrm{O}-1 \\
\mathrm{O} / / \mathrm{H}-5,-\mathrm{O} / \mathrm{O}-4 \\
-\mathrm{H} / / \mathrm{O}-1\end{array}\right.$ & $\left.\begin{array}{l}+,+ \\
+,- \\
-\end{array}\right\}$ & 71.4 & & 71.0 \\
\hline 4 & 69.6 & adj. $3,-\mathrm{O} / \mathrm{O}-3$ &,+- & 70.4 & & 69.9 \\
\hline 5 & 71.9 & $\mathrm{H} / / \mathrm{O}-3$ & + & 67.3 & & 67.9 \\
\hline 6 & 62.5 & 0 & 0 & 62.0 & & 61.2 \\
\hline $\mathrm{CH}_{3}$ & 56.3 & & & 56.3 & & \\
\hline
\end{tabular}

* For assignment $\left(\mathrm{CS}_{2}\right.$ as $\delta$ 193.5) and interpretation, see reference 12 .

The non- ${ }^{1} \mathrm{H}$-decoupled ${ }^{13} \mathrm{C}$-NMR spectrum of BLM A2'-c enhanced by gated decoupling showed that the ${ }^{13} \mathrm{C}-{ }_{-}^{1} \mathrm{H}$ coupling constant of $\mathrm{C}_{1}-\mathrm{H}$ of the mannose moiety was $172.9 \mathrm{~Hz}$ and that of the gulose moiety was $170.1 \mathrm{~Hz}$. These values suggested that both glycosidic configurations were $\alpha^{13)}$, which was in accord with the assignment already determined by ${ }^{1} \mathrm{H}-{ }^{1} \mathrm{H}$ coupling constant and Hudson's rule $^{14)}$.

The Remaining Four Methylenes. ( $\delta 32.8,40.0,41.1$ and 47.8)

These are $\mathrm{CH}_{2}$-carbons of II, V and VI- $\alpha$ and $-\beta$. The signals at $\delta 32.8$ and 40.0 existed in tripeptide $\mathrm{S}$ and did not move in the spectra of ring-expanded BLM and depyruvamide BLM. Therefore, they were assigned to VI- $\alpha$ - and $\beta-\mathrm{CH}_{2}$, respectively. Analyzing the spectra of II, V, pseudotripeptide, pseudotetrapeptide A, ring-expanded BLM and depyruvamide BLM, in which $\mathrm{V}-\mathrm{CH}_{2}$ is lacking, the 
signals at $\delta 41.1$ and 47.8 were assigned to $\mathrm{II}-\mathrm{CH}_{2}$ and $\mathrm{V}-\mathrm{CH}_{2}$, respectively.

The Other Six Methines. ( $\delta 43.5,48.4,53.3,57.7,59.9$ and 60.4)

The signal at the highest field ( $\delta 43.5)$ among them was assigned to III- $\alpha-\mathrm{CH}$, which is the only methine without an attached nitrogen atom. It was confirmed by single frequency ${ }^{1} \mathbf{H}$-decoupling of the III- $\alpha-\mathrm{CH}$ proton ( $\delta$ 2.98). The signal at the lowest field ( $\delta$ 60.4) was assigned to V-CH, which is lacking in depyruvamide BLM. The signal at $\delta 59.9$ could be assigned to I- $\alpha-\mathrm{CH}$ from the spectra of tripeptide S and tetrapeptide S. The signal of II-CH should be shifted significantly in the spectra of ringexpanded BLM and depyruvamide BLM and the signal at $\delta 53.3$ could be assigned to this carbon. The signals at $\delta 48.4$ and 57.7 were assigned to III $-\gamma$ and IV- $\alpha$, respectively, based on the spectra of III, IV, pseudotripeptide and pseudotetrapeptide A.

$\underline{\mathrm{sp}^{2} \text {-Carbons }}$

As already mentioned, the carbamoyl carbon resonated at $\delta 158.5$. Seven $\mathrm{sp}^{2}$-carbon signals ascribed to VI $(\delta 170.9,163.4,162.9,149.4,147.5,125.6$ and 119.7$)$ were easily selected together with two carbonyl signals of I ( $\delta$ 172.5) and III ( $\delta$ 178.1) with reference to the spectra of tripeptide S and tetrapeptide S. The seven signals of VI were assigned to the CO, 2, 2', 4, 4', 5' (d)* and d) in ord5(er from low to high magnetic field with reference to the spectra of BLM-acid and thiazole (Spectrum 17 of reference 15$)$ and by single frequency ${ }^{1} \mathrm{H}$-long-range-decoupling at $\alpha$-methylene proton of VI ( $\left.\delta 3.65\right)$ for the assignment of C-2'. The chemical shift of C-2 of VI was most sensitive among the bithiazole carbons to the carbonyl substitution of VI. This may explain the reason for the chemical shift difference $(0.3 \mathrm{ppm})$ between the signal of BLM A2 ( $\delta$ 163.7) and A2'-c ( $\delta 163.4)$ (see Table 2$)$.

The imidazole carbons of IV were assigned to $\delta 118.5$ (C-5, d), 135.0 (C-4) and 137.5 (C-2, d) with reference to the spectrum of histidine (Spectrum 174 of reference 15, in which the assignment of C-2 and C-4 should be reversed). The chemical shifts of the imidazole carbons, especially of the C-4, were sensitive to $\mathrm{pH}$-changes near the $\mathrm{pK}$-value. The chemical shift difference $(0.3 \mathrm{ppm})$ between the C-4 of BLM A2 ( $\delta$ 134.7) and that of A2'-c ( $\delta$ 135.0) can be explained by a slight difference of pH of the measuring solutions (see Table 2).

The four signals ascribed to the 4-aminopyrimidine chromophore of II were selected and assigned [ $\delta 113.0$ (C-5), 152.9 (C-6), 165.2 (C-4) and 165.9 (C-2)] with reference to the spectrum of thiamine (Spectrum 437 of reference 15) and by single frequency ${ }^{1} \mathrm{H}$-long-range-decoupling at the methine proton of the $\beta$-lactam ( $\delta 4.37)$ for the assignment of C-2.

Among the remaining four carbonyl carbons, the chemical shift of carbonyl of $\mathrm{V}$ would be expected to shift in deamide BLM and ring-expanded BLM and is missing in depyruvamide BLM, while the carbonyl of S-CO of II could be expected to shift in ring-expanded BLM and depyruvamide BLM but not in deamide BLM. Therefore, the former was assigned to $\delta 171.6$ and the latter to 176.7 . The carbonyls of IV ( $\delta 169.5)$ and R-CO of II ( $\delta$ 168.3) could be assigned from the spectra of pseudotripeptide and pseudotetrapeptide A.

Thus, every signal of BLM has been assigned by the structual relation of its fragments and derivatives. The chemical shift table (Table 1) thus obtained, which we named " ${ }^{13} \mathrm{C}$-chemical shift map of bleomycin", contains some information on the conformation of BLM. For example, the chemical shifts of I, III, VI and VII moieties of BLM and its derivatives except for BLM-acid and deglyco-BLMacid are almost the same, that is: derivatizations at sugar and $\beta$-lactam moieties do not affect the I,

* Doublet under off-resonance decoupling. 
III, VI and VII moieties. Chemical shifts of II and V moieties of decarbamoyl BLM and iso-BLM are very similar, but slightly different from those of BLM. This suggests that in aqueous solution there exists an interaction between the 3-O-carbamoyl group and the II and V moieties in BLM molecule. Epimerization at the $\beta$-lactam causes a significant shift at C-5 and C- 6 of II and C- 1 and C-5 of mannose. This should be an important information for conformational studies.

The chemical shift map was already used for the structure determination of ring-expanded BLM and depyruvamide BLM ${ }^{11)}$. In ring-expanded BLM six carbons involved in the ring-expansion shifted distinctly. In depyruvamide BLM three carbons ascribed to the $\mathrm{V}$ moiety are missing and chemical shifts of the side chain carbons of II and C-2 of the pyrimidine chromophore shifted significantly. Thus, this map is useful for structural determination of BLM-related antibiotics and unknown BLM derivatives derived by chemical or biological treatments.

\section{Acknowledgement}

The technical assistance of Mr. MASAYA SATOH is gratefully acknowledged.

\section{References}

1) Takita, T.; Y. Muraoka, T. Yoshioka, A. Fuji, K. Maeda \& H. Umezawa: The chemistry of bleomycin. IX. The structures of bleomycin and phleomycin. J. Antibiotics 25: 755 758, 1972

2) Takita, T.; Y. Muraoka, K. Maeda \& H. Umezawa: Chemical studies on bleomycins. I. The acid hydrolysis products of bleomycin A2. J. Antibiotics 21: 79 80, 1968

3) Takita, T.; K. Maeda, H. Umezawa, S. Омотo \& S. Umezawa: Chemistry of bleomycin. III. The sugar moieties of bleomycin A2. J. Antibiotics 22: 237 239, 1969

4) Омото, S.; T. Takita, K. Maeda \& S. Umezawa: Synthesis of methyl 3-O- and 2-O-carbamoyl- $\alpha$-Dmannopyranosides and carbamoyl-group migration between them. Carbohyd. Res. 30: 239 247, 1973

5) Takita, T.; Y. Muraoka, K. Maeda \& H. Umezawa: Selective cleavage of bleomycin. Proc. 8th Symposium of Peptide Chemistry, Osaka. pp. 179 183, 1970

6) Muraoka, Y.; T. Takita, K. Maeda \& H. Umezawa: Chemistry of bleomycin. VI. Selective cleavage of bleomycin A2 by N-bromosuccinimide. J. Antibiotics 25: 185 186, 1972

7) Umezawa, H.; Y. Takahashi, A. Fujin, T. Saino, T. Shirai \& T. Takita: Preparation of bleomycinic acid: hydrolysis of bleomycin $B_{2}$ by a Fusarium acylagmatine amidohydrolase. J. Antibiotics 26: 117 119,1973

8) Nakayama, Y.; M. Kunishima, S. Omoto, T. Takita \& H. Umezawa: Chemistry of bleomycin. XII. Iso-bleomycin A2, a product of carbamoyl group migration. J. Antibiotics 26: 400 402, 1973

9) Muraoka, Y.; H. Kobayashi, A. Fuji, M. Kunishima, T. Fuji, Y. Nakayama, T. Takita \& H. Umezawa: Chemistry of bleomycin. XVI. Epibleomycin. J. Antibiotics 29: 853 856, 1976

10) Umezawa, H.; S. Hori, T. Sawa, T. Yoshioka \& T. Takeuchi: A bleomycin-inactivating enzyme in mouse liver. J. Antibiotics 27: 419 424, 1974

11) Muraoka, Y.; A. FujiI, T. Yoshioka, T. Takita \& H. Umezawa: Chemistry of bleomycin. XVII. Chemical proof for the $\beta$-lactam of bleomycin. J. Antibiotics 30: 178 181, 1977

12) Perlin, A. S.; B. CASU \& H. J. Koch: Configurational and conformational influences on the carbon- 13 chemical shifts of some carbohydrates. Canad. J. Chem. 48: 2596 2606, 1970

13) Bock, K. \& C. Pedersen: A study of ${ }^{13} \mathrm{CH}$ coupling constants in hexopyranoses. J. Chem. Soc., Perkin Trans. II 1974: 293 297, 1974

14) Oмоto, S.; T. Takita, K. Maeda, H. Umezawa \& S. Umezawa: The chemistry of bleomycin. VIII. The structure of the sugar moiety of bleomycin A2. J. Antibiotics 25: 752 754, 1972

15) Johnson, L. F. \& W. C. JAnkowski: Carbon-13 NMR spectra. John Wiley \& Sons. Inc., New York, 1972 